\title{
Surgical intervention on uterine fibromyoma in a country with limited resources: case of the gynecology-obstetrics department of the Communal Medical Centre of Ratoma Conakry - Guinea
}

\author{
Mamadou H. Diallo ${ }^{1 *}$, Ibrahima S. Balde², Amadou D. Diallo ${ }^{3}$, Ousmane Balde ${ }^{1}$, Fatoumata B. \\ Diallo $^{1}$, Mamadou S. Fofana ${ }^{4}$, Alhassane Sow ${ }^{2}$, Alpha B. Barry ${ }^{2}$, Telly $\mathrm{Sy}^{2}$, Namory Keita ${ }^{1}$
}

\author{
${ }^{1}$ Department Gynecology Obstetrics, Donka National Hospital, Teaching Hospital of Conakry-Guinea \\ ${ }^{2}$ Department Gynecology Obstetrics, Ignace Deen National Hospital, Teaching Hospital of Conakry-Guinea \\ ${ }^{3}$ Department of Visceral Surgery, Donka National Hospital, Teaching Hospital of Conakry-Guinea \\ ${ }^{4}$ Department Gynecology Obstetrics, Ratoma Communal Medical Centre Conakry-Guinea
}

Received: 19 March 2020

Accepted: 20 April 2020

\section{*Correspondence:}

Dr. Mamadou H. Diallo,

E-mail: hadydiallo2002@yahoo.fr

Copyright: ( ) the author(s), publisher and licensee Medip Academy. This is an open-access article distributed under the terms of the Creative Commons Attribution Non-Commercial License, which permits unrestricted non-commercial use, distribution, and reproduction in any medium, provided the original work is properly cited.

\begin{abstract}
Background: In developing countries, treatment of uterine fibromyoma is confronted with numerous problems, namely: financial inaccessibility to the proposed treatments, fear of surgery and the weakness of the technical platform. The objectives of the study were to calculate the frequency of uterine fibromyomas, describe the sociodemographic characteristics of patients, identify the main clinical data and to describe the modalities of surgical management.

Methods: It was a mixed descriptive study, cumulative over a period of 5 years (60 months) with data collection in two phases: a 4-year retrospective study from January 1, 2015 to December 31, 2018 and a 1-year prospective study from January 1, 2019 to December 31, 2019.

Results: Authors collected 135 cases of uterine fibromyomas operated on out of a total of 260 cases of gynaecological pathologies, i.e. a frequency of $51.92 \%$. Nulliparous women were the most concerned $(45.18 \%)$, and women who attended school $(60 \%)$ and those who did not attend school (40\%). Women at home and housewives accounted for $42.20 \%$ and $54.07 \%$ respectively. Clinically, the circumstances of discovery were dominated by menometrorrhagia and menorrhagia respectively $77.77 \%$ and $68.14 \%$. The large uterus was the most frequent physical sign found in $96.29 \%$ of cases. Uterine fibromyomas were recorded in $86.6 \%$ of cases in women with genital activity. The operative indications were dominated by the large polymyomatous uterus (64.44\%), followed by hemorrhagic fibroma $(18.52 \%)$ The surgical treatment was conservative in $92.60 \%$. The total hysterectomy was performed in 7.40 . Lethality was $1.4 \%$.

Conclusions: The surgical management of fibroids contrasts conservative treatment (myomectomy) with radical treatment (hysterectomy) with multiple possible approaches (hysteroscopy, vaginal surgery, laparoscopy or laparotomy). In this context, only laparotomy was possible due to lack of equipment. Laparoscopy and hysteroscopy equipment are necessary for less invasive surgery.
\end{abstract}

Keywords: Fibromyoma, Hysterectomy, Myomectomy, Ratoma

\section{INTRODUCTION}

Uterine fibromyomas or myomas or leiomyomas are benign tumors that develop at the expense of the muscle fibers of the uterus. $20-30 \%$ of women over 35 are affected. ${ }^{1}$ Myomas affect $20-25 \%$ of women of childbearing age and are 3-9 times more common in black women than in white women. ${ }^{2}$ The impact of 
fibromyomas on fertility is controversial, but at date, no study has been able to determine the threshold of number, size, or location of fibroma at which the risk is significant. $^{3}$

The exact etiopathogeny of fibroids remains unclear, although many etiological hypotheses are proposed. The hormonal theory is the most logical one, explained by relative or absolute hyperoestrogenicity and the lack of fibroid development after menopause. There is a familial predisposition and factors significantly associated with the growth of fibroids: obesity, under 12 age when first menstruation occurs, nulliparity and infertility. ${ }^{1}$

Generally, well tolerated or latent, the variety of anatomical forms would explain the pluralism of clinical expression modes, $50 \%$ of women are asymptomatic and are incidentally discovered during a routine gynaecological examination, an ultrasound or pelvic imaging technique. Complementary gynaecological means of exploration are ultrasound, hysterosalpingography, hysteroscopy, and magnetic resonance imaging. ${ }^{1}$

The clinical symptomatology is dominated by uterine bleeding (menometrorrhagia), pelvic organ compression and painful manifestations. ${ }^{4}$

Surgical treatment still remains a very important component and it has benefited in recent years from the contribution of new endoscopic techniques, uterine artery embolization and the treatment with focused ultrasound. ${ }^{1}$

In developing countries, treatment is confronted with numerous problems, namely: financial inaccessibility to the proposed treatments, fear of surgery and the weakness of the technical platform. ${ }^{4}$

Although their degeneration remains insignificant, fibroids are nowadays a real public health problem. In Guinea, the annual statistics of two gynaecologyobstetrics departments of the Conakry University Hospital make uterine fibromyomas the first surgical indication for all gynaecological pathologies. ${ }^{5}$ The high frequency of uterine fibromyomas, difficulties associated with their management, and the inadequacy of previous studies on this pathology in the gynaecology-obstetrics department of the Ratoma communal medical center (CMC) motivated the realization of this work.

\section{Objectives}

- Calculate the frequency of uterine fibromyomas operated on in the gynecology-obstetrics department of the MCC in Ratoma.

- Describe the socio-demographic characteristics of the patients concerned.

- Identify the main clinical data of uterine fibroids operated on at the gynecology-obstetrics department of the MCC in Ratoma.
- Describe the modalities of surgical management of uterine fibroids in the gynaecology-obstetrics department of the MCC in Ratoma.

\section{METHODS}

This study took place in the gynaecology-obstetrics department of the Ratoma Com Munal Medical Centre (a level II facility on the health pyramid of our country). It was a mixed study of a descriptive and cumulative type carried out over a 5-year period (60 months) with data collection in two phases including a 4 year (60 months) from January 1, 2015 to December 31, 2018 phase and another 1 year from January 1, 2019 to December 31, 2019 prospective phase.

This study included all patients who underwent uterine fibromyoma surgery during the study period.

\section{Inclusion criteria}

- Authors included in this study all patients who had undergone surgery for uterine fibromyoma and had a complete medical record.

\section{Exclusion criteria}

- Patients operated on in another facility for uterine fibromyoma and admitted for complicated surgery or other reasons; all patients operated on for conditions other than uterine fibromyoma.

This study was based on a certain number of parameters retained following the review of medical records for the retrospective part and the consultation of patients for the prospective part.

\section{Variables concerned three aspects}

\section{The epidemiological aspect}

The frequency and socio-demographic characteristics which associated age, parity, educational level and socioprofessional category,

\section{The clinical aspect}

Including the circumstances of discovery (menorrhagia, menometrorrhagia, increase in the volume of the abdomen, infertility test), the data of the clinical and paraclinical examination, associated pathologies, the phase of genital life.

\section{The therapeutic aspect}

Grouping together the operative indications, the type of operation performed and the evolution. The collection technique consisted of extracting data from the records for the retrospective part (documentary review), interviewing the patient in bed in hospital and observing 
patients for the prospective part. The results were presented in the form of tables expressed as percentages and averages.

\section{Study population}

Authors carried out an exhaustive census of all cases of uterine fibromyomas operated during the study period at the gynecology-obstetrics department of the Ratoma municipal medical center.

This study protocol was approved by the National ethics committee.

\section{RESULTS}

\section{Frequency}

During the study period, authors collected 135 cases of uterine fibromyomas operated on out of a total of 260 cases of gynaecological pathologies operated on at the gynecology-obstetrics department of the MCC in Ratoma, i.e. a frequency of $51.92 \%$.

Table 1: Annual frequency of UF performed at Ratoma MCC.

\begin{tabular}{|lll|}
\hline Year & Number of UF operated & Percentage \\
\hline 2015 & 39 & $28.88 \%$ \\
\hline 2016 & 26 & $19.26 \%$ \\
\hline 2017 & 33 & $24.45 \%$ \\
\hline 2018 & 15 & $11.11 \%$ \\
\hline 2019 & 22 & $16.30 \%$ \\
\hline Total & 135 & $100 \%$ \\
\hline
\end{tabular}

The highest case frequencies were observed in 2015 (28.88\%), 2016 (19.26\%) and in 2017 (24.45\%).

\section{Socio-demographic characteristics}

Age

The average age of this study patients was 37 with extremes of 20 and 54.

The age group most affected by UF was that of 30-39 with a frequency of $49.63 \%$.

The frequency was identical for the 20-29 and 40-49 age groups $(23.70 \%)$. Those aged 50 and over were less affected $(2.97 \%)$.

\section{Parity}

Nulliparous were the most concerned $(45.18 \%)$, followed by primiparous $(24.44 \%)$, pauciparous represented $(15.60 \%)$ followed by multiparous $(13.33 \%)$ and major multiparous $(1.48 \%)$.

\section{Educational level}

The analysis of this parameter shows a higher frequency among educated women $(60 \%)$; however, this rate is not negligible among uneducated women $(40 \%)$.

\section{Socio-professional category}

Housewives were the most affected (42.20\%), followed by women in liberal professions and salaried women with $32.60 \%$ and $20.00 \%$ respectively. Pupils and students accounted for $5.20 \%$.

\section{Marital status}

Married women were the most affected (54.07\%), single women also represented a considerable proportion $(4.0 \%)$.

\section{Clinical features}

Table 2: Distribution of patients by circumstances of discovery.

\begin{tabular}{|lll|}
\hline Circumstances of discovery & $\mathbf{n = 1 3 5}$ & Percentage \\
\hline Momenetrorrhagia & 106 & $\mathbf{7 7 . 7 7 \%}$ \\
\hline Menorrhagia & 92 & $68.14 \%$ \\
\hline Feeling of abdominopelvic mass & 48 & $35.55 \%$ \\
\hline Infertility check-up & 14 & $10.37 \%$ \\
\hline Fortuitous discovery & 13 & $9.62 \%$ \\
\hline Pelvialgies & 11 & $8.14 \%$ \\
\hline $\begin{array}{l}\text { Increase in the volume of the } \\
\text { abdomen }\end{array}$ & 9 & $6.67 \%$ \\
\hline Pollakiurie & 7 & $5.18 \%$ \\
\hline Miscarriages & 5 & $3.70 \%$ \\
\hline
\end{tabular}

The circumstances of discovery were dominated by the menometrorrhagia followed by menorrhagia, a sensation of abdominopelvic mass and infertility assessment.

One patient had 2 or more functional signs.

Table 3: Patients distribution according to gynaecological examination data.

\begin{tabular}{|lll|}
\hline $\begin{array}{l}\text { Gynaecological } \\
\text { examination data }\end{array}$ & $\mathbf{n = 1 3 5}$ & Percentage \\
\hline Large uterus & 130 & 96.29 \\
\hline Genital hemorrhage & 98 & 72.59 \\
\hline Apparently normal & 5 & 3.70 \\
\hline $\begin{array}{l}\text { Polyp delivered through the } \\
\text { cervix }\end{array}$ & 14 & 10.37 \\
\hline Exo cervicitis & 12 & 8.88 \\
\hline Adnexal mass & 7 & 5.18 \\
\hline
\end{tabular}

The large uterus and genital hemorrhage were the most common signs. 
Only one patient had 2 or 3 functional signs.

Ultrasound was performed in most cases and hysterosalpingography more rarely.

Table 4: Distribution of patients according to whether or not additional diagnostic tests were performed.

\begin{tabular}{|lll|}
\hline Additional diagnostic tests & $\mathbf{n = 1 3 5}$ & Percentage \\
\hline Abdominopelvic ultrasound & 133 & $98.51 \%$ \\
\hline Hysterosalpingography & 7 & $5.18 \%$ \\
\hline Nothing & 2 & $1.48 \%$ \\
\hline
\end{tabular}

Table 5: Distribution of patients by topographic shape of fibromyomas on ultrasound (FIGO classification).

\begin{tabular}{|lll|}
\hline FIGO classification & $\mathbf{n = 1 3 5}$ & Percentage \\
\hline Type 0 & 40 & $29.62 \%$ \\
\hline Type 1 & 28 & $20.74 \%$ \\
\hline Type 2 & 34 & $25.18 \%$ \\
\hline Type 3 & 29 & $20.75 \%$ \\
\hline Type 4 & 17 & $12.59 \%$ \\
\hline Type 5 & 18 & $13.33 \%$ \\
\hline Type 6 & 16 & $11.85 \%$ \\
\hline Type 7 & 13 & $9.62 \%$ \\
\hline Type 8 & 14 & $10.37 \%$ \\
\hline
\end{tabular}

Type 0 of the FIGO classification was the most encountered $(29.62 \%)$ followed by type $2(25.18 \%)$ and 3 $(20.75 \%)$.

Existence of a gynaecological pathology associated with ultrasonography. Based on the ultrasound examination, the fibroid was associated with gynaecological pathology in $11.1 \%$ of cases. It was an ovarian cyst in 12 cases $(8.88 \%)$ and hydrosalpinx in 3 cases $(2.22 \%)$.

\section{Genital life phase}

Uterine fibromyoma was recorded in $86.6 \%$ of cases in women during genital activity, in $10.37 \%$ in perimenopausal women and in $2.97 \%$ in postmenopausal women.

\section{Surgical management}

\section{Patient distribution by surgery indication}

Surgical indications were dominated by the large polymyomatous uterus $(64.44 \%)$, followed by the hemorrhagic fibroma $(18.52 \%)$ and infertility which accounted for $17.04 \%$.

Distribution of patients according to the surgical procedure performed.

The surgical treatment was conservative in 125 cases $(92.60 \%)$ and consisted of a simple myomectomy and/or polymyomectomy. The whole hysterectomy was performed in 10 cases (7.40\%). Associated lesions were also managed simultaneously (cystectomy, hydrosalpinx cure).

\section{Results of the anatomopathological examination}

Out the 135 surgeries performed for uterine fibromyoma, 80 pieces $(59.25 \%)$ were examined with confirmation of the diagnosis of leiomyoma in all cases, while uterine fibromyoma was associated with endometrial hyperplasia in 7 cases of hysterectomy (5.18\%), an ovarian cyst in 12 $\operatorname{cases}(8.88 \%)$ and a cervical cancer in one extra-facial hysterectomy case $(0.74 \%)$.

\section{Prognosis}

\section{Per and postoperative complications}

Intraoperative complications were dominated by haemorrhage, which affected 35 patients $(25.92 \%)$, with $65.18 \%$ of post-operative complications being uncomplicated and $34.82 \%$ complicated. These complications were dominated by anaemia (haemoglobin level lower than $10 \mathrm{~g} / \mathrm{dl}$ ) in 32 patients $(23.70 \%)$ whose myomectomy was haemorrhagic and required a blood transfusion in 21 cases $(27.15 \%)$.

Authors recorded 2 death cases due to severe anemia complicated by hypovolemic shock, i.e. a case-fatality rate of $1.48 \%$.

\section{DISCUSSION}

\section{Frequency}

The highest frequencies of cases observed in 2015, 2016 and in 2017 are linked to the closure of the Donka National Hospital for renovation, resulting in a considerable increase in consultations at the Ratoma municipal medical center.

Study frequency is higher than those reported by some authors, notably: Mahbouli S et al, in Tunisia reporting a rate of $19.1 \%$ in relation to all gynaecological surgery and Laghzaoui $\mathrm{M}$ et al, in Morocco reporting a rate of $15 \%$ in relation to all gynaecological pathology. ${ }^{6,7}$ On the other hand, this frequency is lower than those reported by other authors, notably Dia A et al who reported a rate of $58.62 \%$ of all gynaecological disorders operated on. ${ }^{8}$ Sy $\mathrm{T}$ et al in Guinea reported a frequency of $49.92 \% .{ }^{5}$ Baldé IS et al reported a frequency of $42.4 \%$ at the Conakry University Hospital. ${ }^{4}$

Study high frequency could be explained by the fact that the study took place in a level II reference centre in the health pyramid of our country, which is easily accessible and the cost of the intervention affordable. This frequency is often underestimated because it only takes into account operated uterine fibromyomas which are 
symptomatic as described by Cramer and Patel (quoted by Razafindrabe JA). ${ }^{9}$ On a serial anatomopathological study of 100 consecutive hysterectomies for usual pathologies, the incidence of uterine fibromyomas rose from $33 \%$ of preoperative diagnosis to $77 \%$ after an anatomopathological study (less than $1 \mathrm{~cm}$ ).

\section{Socio-demographic characteristics}

Concerning age, this study result is different from those described in the literature: 43 years according to Parazzini quoted by Mahbouli; 40-49 years according to Dia et al, in Senegal, i.e. $43.6 \% .^{6,8}$ In this series, a rate of $23.70 \%$ was recorded for the 20-29 age group, which reinforces the idea that in black women, uterine fibromyomas are increasingly found in young people.

Regarding parity, study result is different from that of some authors, notably Mahbouli S et al in Tunisia who report similar rates between nulliparous (30.6\%), pauciparous $(31.5 \%)$ and multiparous $(37.9 \%){ }^{6}$

Baldé IS et al, in Guinea reported a rate of $32.8 \%$ for nulliparous and $23.8 \%$ for pauciparous. ${ }^{4}$ This difference could be explained by the difference between the physioanatomical data of black women and those from the Maghreb. However, the notion reported by ROSS et al, that the relative risk of having a uterine fibromyoma decreases with parity is not unanimous. ${ }^{10}$ The high frequency in nulliparous women could be explained by the relationship between infertility and uterine fibromyomas, due to the climate of hyperoestrogenism which could be at the root of this phenomenon which has a socio-cultural importance especially in developing countries where societies advocate pro-natalist behaviour.

With regard to the level of education, this study findings are consistent with those of Baldé IS et al in Guinea, who reported a rate of $54.8 \%$ among educated women and $45.2 \%$ among unschooled women, respectively. ${ }^{4}$ There is no proven relationship between the occurrence of uterine fibromyoma and a woman's level of education. However, educated women have more access to health care than uneducated women, they use contraceptives and often marry late, so they are more exposed to the effects of estrogen. The high frequency of housewives in this series could be explained by the fact that they make up the bulk of the female population in Guinea. ${ }^{11}$

\section{Clinical features}

This study results are similar to those of Laghzaoui $\mathrm{M}$ et al in Morocco reporting that the functional signs of uterine fibromyomas were dominated by genital hemorrhages $(68.26 \%)$ and these hemorrhages were represented essentially by menorrhagia ( $68.3 \%$ of cases). ${ }^{6}$ On the other hand, Dia A et al, in Senegal reported that the increase in the volume of the abdomen was the most frequent reason for consultation (96.4\% of cases). ${ }^{8}$ This difference could be explained by the difference in vocation between the study services (surgery in Senegal and gynecology-obstetrics in Senegal).

Patients with an abdominopelvic mass are more likely to use surgery than gynecology services and conversely for those with genital bleeding. The predominance of large uterus and genital haemorrhage has also been reported in the Baldé IS and Razanfindrabe JA et al studies. ${ }^{4,9}$ The same is true for pathologies associated with uterine fibromyoma.

Additional diagnostic tests were performed in $98.51 \%$ of cases. Among these patients all benefited from abdominal pelvic ultrasound, first-line examination and 7 benefited from hysterosalpingography (5.18\%). Magnetic resonance imaging and hysteroscopy were not performed due to the lack of equipment. Dia A et al, in Senegal reported that abdominopelvic ultrasound was performed in $42.28 \%$ of cases and hysterosalpingography in $12.14 \%{ }^{8}$

Contrary to Lagzaoui $\mathrm{M}$ et al, reporting the realization in $100 \%$ of the cases in his series. ${ }^{7}$ The closer one is to menopause the more the number of uterine fibromyomas decreases, the same observations were made in the study of Mahbouli $\mathrm{S}$ et al, in Tunisia reporting in their series $75 \%$ of women in genital activity period; $14.6 \%$ in the perimenopausal period and $10 \%$ of women in confirmed menopause in whom the age of menopause appears to be delayed (51.25 years). ${ }^{6}$

With regard to the existence of a gynaecological pathology associated with the fibroid, this study observation is related to Célui de Laghzaoui $\mathrm{M}$ et al, in Morocco who reported in his series that according to ultrasound, uterine fibroid was associated in 24 cases with a cyst of the ovary and a hydrosalpinx in 17 cases. $^{7}$

\section{Surgical management}

Concerning the operative indication, this study observation is shared by Langzaoui $\mathrm{M}$ et al in Morocco, reporting in his series that the operative indications were dominated by large uterine fibroids (41\%) and large polymyomatous uteri $(38.16 \%) .^{7}$ Therapeutic intervention must be motivated by the existence of an embarrassing symptom and only clinical surveillance is necessary in the case of asymptomatic fibroma. A possible future desire for pregnancy must be taken into account as well as the age of occurrence of menopause. ${ }^{12}$

The choice of myomectomy and hysterectomy was guided by several factors, of which age and parity were the most important. The size of the uterus and anatomical features were rather decisive in the choice of the surgical approach. Myomectomy was suggested for young women and those who wish to become pregnant in the absence of pregnancy. This choice is still not possible, especially when there are many fibroids, and there is a risk that after myomectomy a fibrous stunted uterus may be left behind, 
sometimes with tubo-ovarian adhesion sclerosis, which may compromise reproductive function and which is not safe from recurrence.

This study finding is different from that of Dia A et al in Senegal, who reported more hysterectomies in his series: $35.72 \%$ subtotal and $27.86 \%$ total hysterectomy, with a myomectomy rate performed on $43 \%$ of cases in his series, performed by three routes: abdominal (80.8\%), hysteroscopic (13.8\%) and laparoscopic (5.3\%). ${ }^{8}$ Authors did not perform hysteroscopy and laparoscopy due to the lack of equipment.

\section{Prognosis}

Anaemia was found in the study by Mahbouli $\mathrm{S}$ et al, in Tunisia reporting 54\% with a hemoglobin level under 12 g/dl. ${ }^{6}$ Baldé IS et al also found anaemia in their series. ${ }^{4}$ Dia A et al in Senegal reported $0.7 \%$ deaths, while Mahbouli et al in Tunisia reported no deaths in their series of 219 patients. ${ }^{6}$

Data in the literature reported a lethality lower than $1 \% .^{13,14}$

Study high lethality rate $(1.48 \%)$ is thought to be related to the lack of resuscitation equipment.

Limitations and difficulties of the study encountered problems were incomplete records, inadequacy in the summary description of results: in particular the precision of the location and ultrasound characteristics of the myomas observed. The lack of equipment for hysteroscopy.

\section{CONCLUSION}

The frequency of uterine fibroids is important in gynaecological surgery; signs of discovery of myomas that may point to a surgical solution may be menorrhagia, metrorrhagia or menometrorrhagia most often leading to iron deficiency anaemia, pelvic pain or heaviness, compression of neighbouring organs (urinary, digestive or vascular). The surgical management of fibroids opposes the conservative treatment (myomectomy) with the radical treatment (hysterectomy) with multiple possible approaches (hysteroscopy, vaginal surgery, laparoscopy or laparotomy).

In study context only laparotomy was possible due to a lack of equipment. The surgical indications vary from one patient to another and must take into account the desire for fertility, the psychology of the patient, the surgical risks and the medium and long-term consequences of the operation. It is important, in the context of myomatous pathology, to surgically treat only fibroids that are symptomatic or may affect fertility.

The objectives of surgical treatment are multiple, namely the improvement of symptoms caused by fibroids, the improvement of fertility, and in some cases the prevention of serious complications inherent to fibroids. The surgical treatment of fibroids is presented as a curative solution as opposed to medical treatment, which is usually only symptomatic. Surgical proposals must always integrate patients' wishes, such as the desire for pregnancy, the desire to preserve the uterus or the ovarian function (pre-menopause, peri-menopause, menopause).

Funding: No funding sources

Conflict of interest: None declared

Ethical approval: The study was approved by the Institutional Ethics Committee

\section{REFERENCES}

1. National College of French Gynecologists and Obstetricians (CNGOF): Abstract of GynecologyObstetrics, $2^{\text {nd }}$ edition Masson, Paris; 2011:227-228.

2. Okogbo FO, Ezechi OC, Loto OM, Ezeobi PM. Uterine leimyomatan in South Western Nigeria: a clinical study of presentations and management outcome. Afr Health Sci. 2011;11(2):271-8.

3. Bajekal N, Li TC. Fibroids, infertility and pregnancy wastage. Hum Report Update; 2000;6:614-20.

4. Baldé IS, Diallo BS, Conté I, Baldé O, Diallo MH, Diallo BC, et al. Uterine fibromyomas: epidemiological and clinical aspects, surgical management and prognosis at Conakry University Hospital Rev int sc méd-RISM6. 2015;17(3):118-24.

5. Sy T, Diallo Y, Diallo AB, Diallo FB, Touré A, Keita N, Diallo MS. Uterine fibromyomas: epidemioclinical aspect and surgical management at the Ignace Deen gynecology-obstetrics clinic of the CHU of Conakry. Ann Univer Ouagadougou- série D. 2007;5:113-26.

6. Mahbouli S, Messaoudi Y, Chandoul Y, Zayene H, Messaoudi F, Basly M, et al. management of uterine fibroids (about 219 cases), Tunisie Méd. vol 79- $\mathrm{N}^{\circ}$. 2001;10:515-20.

7. Laghzaoui M, Boukaidi S, Bouhya S, Hermas S, Bennani O, Aderdour M. epidemiology of uterine fibroids (about 690 cases). Maroc Med. 2001;23(4):266-70.

8. Dia A, Beye SB, Dangou JM, Dieng M, Woto Gaye CT. Uterine fibroids at the Dakar University Clinic: about 140 cases operated on in two years Dakar. Méd. 2003;48(2):72-6.

9. Razafindrabe JA, Rabarijaona M, Rakotoarisoa B, Radriamaro B, Sambany R, Razafindramboah: reasons for hysterectomy in a Gynecology and Obstetrics department. Med. Afr Nre. 2002;49(4):166-8.

10. Ross RK, Pike MC, Vessey MP. Risk factors for uterine fibroids: reduced risk associated with oral contraceptives. Br Med J. 1986;293:359-62.

11. Diallo MDD, Bussangu MF. Archive of the Ministry of Planning, National Institute of Statistics, Conakry, Guinea: Demographic and Health Survey (DHS). 
Guinea, Calvelton, Maryland, USA: INS and ORC Marco; 2012:246-247.

12. Bezard F. uterine fibromyoma: treatment. Drug treatments for uterine fibroma. Argumentaire. 2001;14:433-45.

13. Abbara A. Uterine fibromyoma: generalities. J Gynecol Obstet Biol Reprod. 2004;42:716-9.

14. Zhioua F, Ferciou M, Mouelhi C. Contribution of operative hysteroscopy in the treatment of intrauterine myomas in infertile patients. Magreb Med. 1997;320:34-6.

Cite this article as: Diallo MH, Balde IS, Diallo AD, Balde O, Diallo FB, Fofana MS, et al. Surgical intervention on uterine fibromyoma in a country with limited resources: case of the gynecology-obstetrics department of the Communal Medical Centre of Ratoma Conakry - Guinea. Int J Reprod Contracept Obstet Gynecol 2020;9:2227-33. 